\title{
Effect of Cavitation Modelling on the Prediction of the Lubrication Performance Using CFD: A Case Study of Journal Bearing Lubricated with Non-Newtonian
}

\author{
M. Tauviqirrahman ${ }^{\# 1}$, B. Muthik ${ }^{\#}$, M. Muchammad ${ }^{* 2}$, A.W. Pratomo ${ }^{\#}$, J. Jamari ${ }^{\#}$

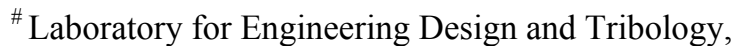 \\ Department of Mechanical Engineering, Diponegoro University \\ Jl. Prof. Soedharto, Tembalang, Semarang, Indonesia, Phone: 024-7460059 \\ ${ }^{1}$ mohammad.tauviqirrahman@ft.undip.ac.id \\ * Laboratory for Surface Technology and Tribology, \\ Faculty of Engineering Technology, University of Twente \\ Drienerloolaan 5, Postbus 217, 7500 AE, Enschede, The Netherlands \\ 2m_mad5373@yahoo.com
}

\begin{abstract}
The effect of cavitation on the lubrication performance of the journal bearing operating with non-Newtonian lubricant is studied. The non-Newtonian behaviour of lubricant is addressed based on power law fluid model. The hydrodynamic performance in terms of pressure distribution and load carrying capacity and how it depends on power law index are explored using CFD (computational fluid dynamic). The comparison between the case of "no-cavitation" and the "with cavitation" for the lubrication analysis is also performed. It is found that including the cavitation modelling in the analysis gives a more accurate prediction with respect to the load carrying capacity and vortex development. In addition, more significant error between the case of 'no cavitation' and the case of 'with cavitation' is found when $n=1$ (i.e. Newtonian lubricant).
\end{abstract}

Keyword- Cavitation; CFD (Computational Fluid Dynamic); lubrication; non Newtonian.

\section{INTRODUCTION}

Journal bearing is mechanical element designed to carry shaft or journal which rotates freely in a supporting metals or shell. The insertion of a lubricant into the region around the interacting devices could avoid direct contact between the surfaces, so the wear can be minimized. In addition, the pressure gradients that occur in the clearance between the journal and the bearing yield the load-carrying capacity. Due to the divergent-convergent gap, numerically the analysis of hydrodynamically lubricated journal bearings falls into several classes, using the Sommerfeld theory, using Half-Sommerfeld theory, using Reynolds approach, or using mass-conservative approach. The first three theories are often considered as a rough approximation, because it is not based on real physical phenomenon [1].

There are few numerical works performed in literature to address the bearing lubricated by non-Newtonian either by taking into account the cavitation model or not. Mishra [2] proposed a numerical method by solving the Reynolds equation and energy equation to investigate the effect of journal misalignment, bore noncircularity, and lubricant non-Newtonian behaviour. In order to describe the lubricant characteristic in the cavitation region, Elrod method [3] was adopted. Yadav and Sharma [4] analysed the influence of the texturing parameter on the static and dynamic performance characteristic of the bearing. Lin et al. [5] studied the nonNewtonian effects on the non-linear stability boundary of short journal bearings through the transient non-linear analysis. Pratomo et al. [6] investigated the combined effect of single texturing (pocketing) and wall slip on pressure. The modified Reynolds equation for lubrication with non-Newtonian power-law fluid was proposed. It was shown that the maximum load support is achieved when the pocket depth is equal to the film thickness. However, in their study, the cavitation Reynolds boundary condition was applied. In the following work, Tauviqirrahman et al. [7] using a mass conserving numerical approach for modelling cavitation investigated hydrodynamic lubrication performance of single-textured bearing. In recent lubrication, Khatri and Sharma [8] presented a comparative study between textured surface and non-textured surface non-recessed hybrid journal bearing configurations. However, in their study, the analysis was performed based on Reynolds theory which may be questionable.

Based on survey literature, one can find that the researches about journal bearing with non-Newtonian lubricant considering cavitation are very rare. Starting from this point, understanding that the cavitation has a huge influence on the analysis of the journal bearing, a numerical technique based on CFD (computational fluid dynamic) model is proposed. The effect of cavitation modelling on the lubrication behaviour (pressure 
distribution, streamline) varying different lubricant conditions is also examined. It will be shown that neglecting the cavitation model in the analysis leads to the overestimation of the predicted hydrodynamic performance.

\section{NUMERICAL ANALYSIS}

\section{A. Governing Equations}

In the present study, the analysis of lubrication is solved by the Navier-Stokes equations. The Navier-Stokes equations are solved over the domain using a finite-volume method with the commercial CFD software package FLUENT®. The equations are applied with constant density and viscosity, without body force. The equations are steady and solved in the $x$ - and $z$-direction only. With these properties the Navier-Stokes and the continuity equations can be expressed, respectively,

$$
\begin{gathered}
\rho(\mathbf{u} \bullet \nabla) \mathbf{u}=-\nabla p+\eta \nabla^{2} \mathbf{u} \\
\nabla \bullet \mathbf{u}=0
\end{gathered}
$$

In the present study, the shear stress-strain relation of lubricant is modelled with the scheme of a power-law fluid model. It means that the shear stress $\tau$ is a function of some power of shear strain rate $\dot{\gamma}$ and its mathematical expression reads [8]:

$$
\tau=m(\dot{\gamma})^{n}
$$

where $m$ and $n$ are consistency and flow behaviour index, respectively.

For more representative of the bearing characteristic, the flow is considered turbulent. The turbulent model of Realizable $k-\varepsilon$ is used with standard wall functions as near-wall treatment. In the journal bearing, when the flow enters the divergent region, pressure might fall below the saturation vapor pressure, and the liquid would rupture and the cavitation occurs. In FLUENT ${ }^{\circledR}$, there are three available cavitation models: Schneer and Sauer model, Zwart-Gelber-Belamri model and Sighal et al. model [9]. In this study, the Zwart-Gelber-Belamri is employed due to their capability (less sensitive to mesh density, robust and converge quickly [9]).

In cavitation, the liquid-vapor mass transfer (evaporation and condensation) is governed by the vapor transport equation [9]:

$$
\frac{\partial}{\partial t}\left(\alpha_{v} \rho_{v}\right)+\nabla \cdot\left(\alpha_{v} \rho_{v} \mathrm{v}\right)=R_{g}-R_{c}
$$

where $\alpha_{v}$ is vapour volume fraction and $\rho_{v}$ is vapor density. $R_{g}$ and $R_{c}$ account for the mass transfer between the liquid and vapour phases in cavitation. For Zwart-Gelber-Belamri model, the final form of the cavitation is as follow [10]:

$$
\begin{aligned}
& \text { If } p \leq p_{v}, R_{g}=F_{\text {evap }} \frac{3 \alpha_{\text {nuc }}\left(1-\alpha_{v}\right) \rho_{v}}{R_{\mathrm{B}}} \sqrt{\frac{2}{3} \frac{\mathrm{P}_{v}-\mathrm{P}}{\rho_{\ell}}} \\
& \text { If } p \geq p_{v}, R_{c}=F_{\text {cond }} \frac{3 \alpha_{v} \rho_{v}}{R_{\mathrm{B}}} \sqrt{\frac{2}{3} \frac{\mathrm{P}-\mathrm{P}_{v}}{\rho_{\ell}}}
\end{aligned}
$$

where $F_{\text {evap }}=$ evaporation coefficient $=50, F_{\text {cond }}=$ condensation coefficient $=0.01, R_{B}=$ bubble radius $=10^{-6} \mathrm{~m}$, $\alpha_{\mathrm{nuc}}=$ nucleation site volume fraction $=5 \times 10^{-4}, \rho_{l}=$ liquid density and $p_{v}=$ vapour pressure.

\section{B. CFD Model}

Geometry of journal bearing and its schematic representation are shown in Table I and Fig. 1, respectively. The journal bearing consists of two conformal surfaces (shaft and housing). By simulation, the computational domain is bounded by two surfaces: the cylindrical surface of the journal and the cylindrical surface of the bearing. For both surfaces, the no-slip boundary condition is employed. The boundary corresponding to the bearing is steady, which means that the outer surface is modelled as a stationary wall, while the internal cylindrical surface is set into rotation by imposing a rotational velocity. The mesh is built using the standard mesh feature provided by ANSYS. This is a mesh generator that enables the construction of a mesh automatically. Based on the mesh refinement study, it is found that 2100 elements are sufficient to get the desired level of accuracy for the bearing. In the present study, power law index $\mathrm{n}$ is varied: $0.5,0.75$, and 1 . The density of the fluid $\rho$ is $960 \mathrm{~kg} / \mathrm{m} 3$, while consistency index $m$ is 0.04 . 
TABLE I. Properties of the Studied Bearing

\begin{tabular}{|c|c|c|c|c|}
\hline \multicolumn{2}{|c|}{ Diameter (mm) } & \multirow{2}{*}{$\begin{array}{l}\text { Clearance } \\
\quad(\mathrm{mm})\end{array}$} & \multirow{2}{*}{$\begin{array}{l}\text { Eccentricity } \\
\text { (mm) }\end{array}$} & \multirow{2}{*}{$\begin{array}{l}\text { Rotational speed } \\
\text { (rpm) }\end{array}$} \\
\hline Journal & Bearing & & & \\
\hline 42.7 & 50 & 0.2 & 3.4 & 120 \\
\hline
\end{tabular}

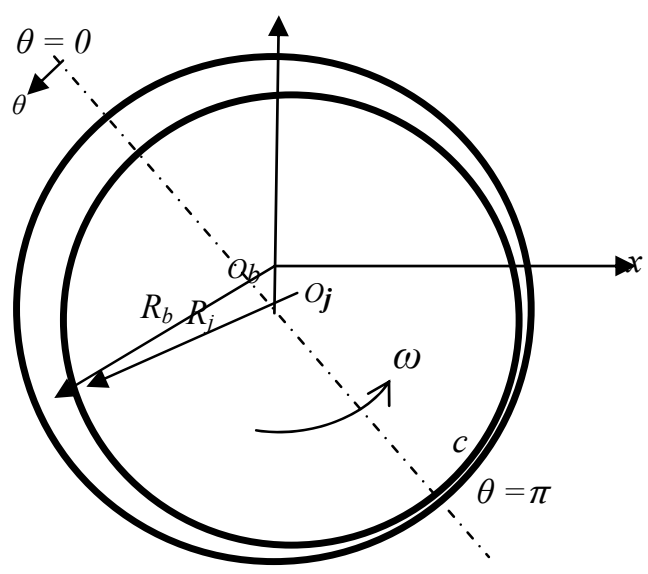

$c=$ Clearance

$R_{b}=$ Radius of bearing

$R_{j} \quad=$ Radius of journal

$O_{b}=$ Centre of bearing

$O_{j}=$ Centre of journal

$\omega=$ Radial speed

$\theta=$ Angular coordinate

Fig. 1. Schematic representation of journal bearing

\section{III.RESULTS AND DISCUSSIONS}

Results are presented in terms of fluid film pressure and streamlines. The interest main in this paper is thus to analyze the cavitation effects in hydrodynamic performance. The hydrodynamic pressure and the load carrying capacity are of main particular interest. It should be noted that the load carrying capacity is defined by integrating the calculated hydrodynamic pressure field along the surface contact. However, other effects such as mechanical feature and thermodynamics would certainly influence the hydrodynamics. In the present study, the comparison between the analysis considering cavitation modeling and the analysis without cavitation is conducted.

\section{A. Pressure Distribution}

Comparisons of pressure distribution for journal bearing are made in Fig. 2, where $p$ is plotted as a function of the spatial dimension $\theta$ for different values of $n$. Obviously, as can be seen from Fig. 2 , there is an increase in the hydrodynamic pressure distribution as well as their peak with increasing the power law index $n$. It indicates that on the other words, the maximum and minimum oil pressures are affected by the viscosity characteristic. This trend prevails both for the case of "with cavitation" and the case of "no-cavitation".

Fig. 2 also shows that when the cavitation modelling is included in the analysis, a significant effect is observed, that is, the predicted pressure is always smaller than that without cavitation for all values of $n$. With respect to the peak hydrodynamic pressure, it is also observed from Fig. 2 (c) that for $n=1$ (i.e. Newtonian fluid), there is a big discrepancies of the predicted result between the case of "cavitation" and "no cavitation". It is up 79\%. However, regarding with the load carrying capacity, the discrepancy between two kinds of analysis considered here is just around $23 \%$. Based on the physical point of view, for case of "no cavitation" which means that Eqs. 4-6 are not solved, the change of phase from liquid to vapour which may occur in the divergent region of the journal bearing representing the cavitation phenomena is not modelled. This will be different if the cavitation model is considered in the analysis (i.e. the case of "with cavitation"). Based on the numerical point of view, it indicates that including the cavitation modelling is very important in the analysis, especially for Newtonian fluid. The results subject to the hydrodynamic pressures and the corresponding load carrying capacities for different values of $n$ are shown Table II. Based on Table II, it can be observed that the prediction of the lubrication behaviour without considering cavitation is around 10 to $80 \%$ larger than that with cavitation. In general, these numerical results can be comparable with the work of Qiu and Khonsari [11] in the case of fully textured rotating thrust bearing. They confirmed that a non-mass-conserving treatment of cavitation leads to a significant overestimation of load carrying capacity. 


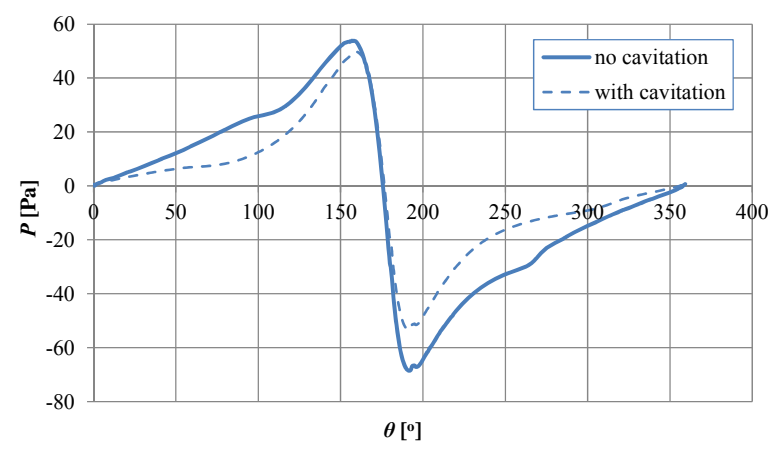

(a)

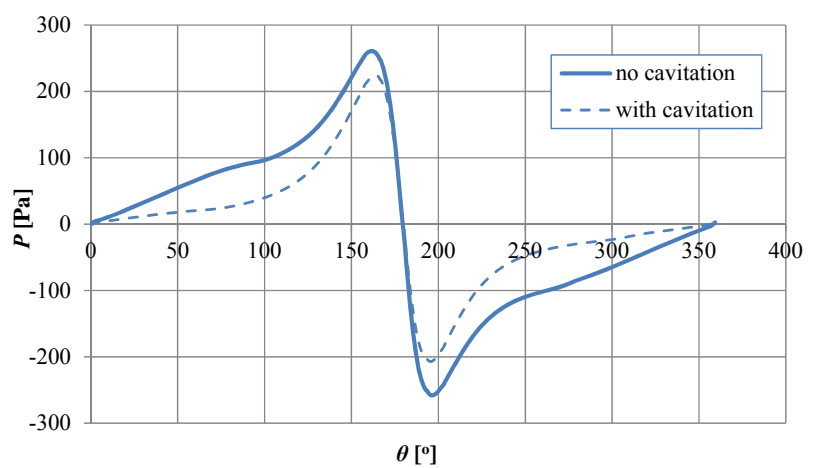

(b)

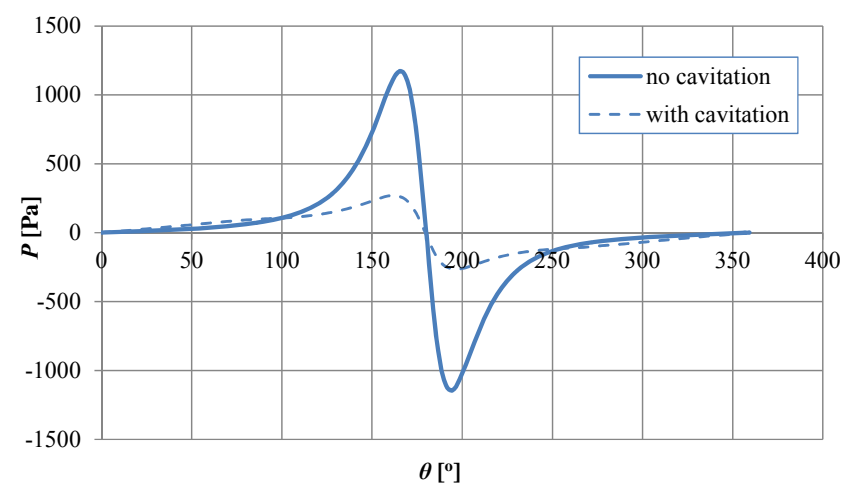

(c)

Fig. 2. Circumferential pressure distributions both for analysis with cavitation and without cavitation for (a) $n=0.5$; (b) $n=0.75$; (c) $n=1$.

TABLE II. Lubrication Performance of the Bearing Studied Here

\begin{tabular}{|c|c|c|c|c|c|c|}
\hline \multirow{2}{*}{$n$} & \multicolumn{3}{|c|}{ Peak pressure $P_{\max }(\mathrm{Pa})$} & \multicolumn{3}{c|}{ Load carrying capacity $W(\mathrm{~N})$} \\
\cline { 2 - 7 } & $\begin{array}{c}\text { no } \\
\text { cavitation }\end{array}$ & $\begin{array}{c}\text { with } \\
\text { cavitation }\end{array}$ & $\begin{array}{c}\text { Deviation } \\
(\%)\end{array}$ & $\begin{array}{c}\text { no } \\
\text { cavitation }\end{array}$ & $\begin{array}{c}\text { with } \\
\text { cavitation }\end{array}$ & $\begin{array}{c}\text { Deviation } \\
(\%)\end{array}$ \\
\hline 0.5 & 50 & 45 & 10 & 0.27 & 0.24 & 11 \\
\hline 0.75 & 250 & 220 & 12 & 0.74 & 0.63 & 14 \\
\hline 1 & 1200 & 250 & 79 & 1.00 & 0.77 & 23 \\
\hline
\end{tabular}

\section{B. Streamline}

Streamlines can give a qualitative idea about how the lubricant behaves if there are any vortexes or irregularities. Streamlines are expressed in terms of the scalar stream function $\psi$ and along a streamline $\psi=$ constant. Derivations of $\psi$ refer to the corresponding velocities, i.e. $u=\partial \psi / \partial y$ and $v=\partial \psi / \partial x$.

In Fig. 3, stream functions for bearing geometry are plotted with different power law index $n$. In the case of "with cavitation", Fig. 3 (a) and (b) shows the same vortex development. While in Fig. 4 corresponding the $n$ of 
1 (i.e. Newtonian lubricant), the vortex develops from $\theta$ of $230^{\circ}$ to $260^{\circ}$. Based on the physical point of view, the level of power law strongly affects the behavior of lubricant. The higher the $n$, the smaller the vortex development. On the other words, increasing the $n$ up to the Newtonian level will reduce the cavity flow phenomenon. It is interesting to note that when the cavitation modeling is excluded in the analysis, the simulation depicts the same vortex development (i.e. same size and location of the vortex) for all values of $n$ as shown in Fig. 5 (a) and (b). It means that the variation of $n$ seems to be nothing regarding their influence on the vortex. And of course, it becomes less accurate if the cavitation modeling is not taken into account. By studying Fig. 3, the conclusion can be drawn that the vorticity is clearly dependent on the cavitation.

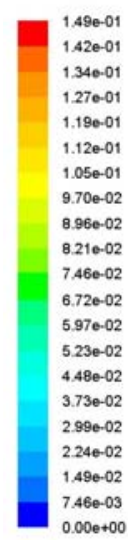

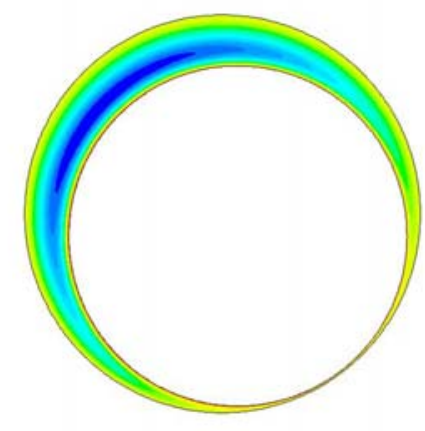

(a)

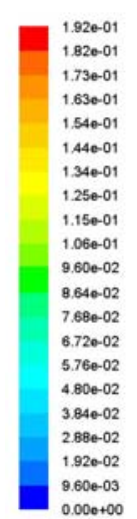

$000+00$

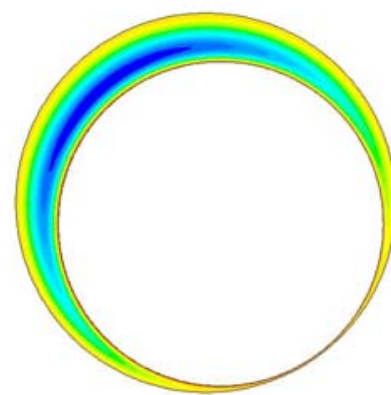

(b)

Fig. 3. Stream function in the bearing in the case of "with cavitation" for two values of power law index (a) $n=0.5$, (b) $n=0.75$

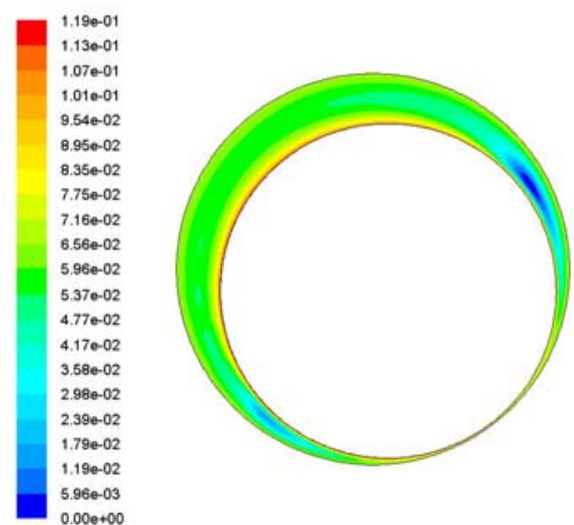

Fig. 4. Stream function in the bearing in the case of "with cavitation" for $n=1$

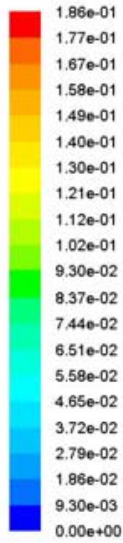

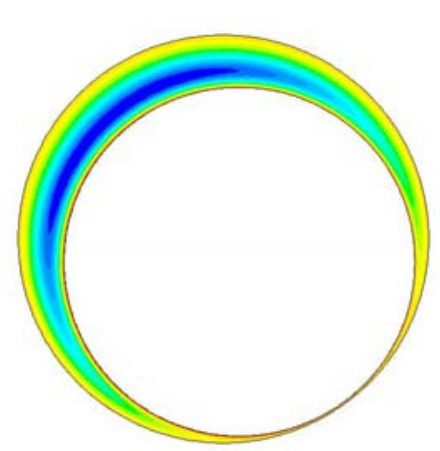

(a)

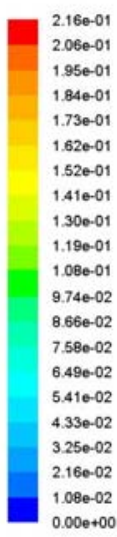

(b)

Fig. 5. Stream function in the bearing in the case of "without cavitation" for two values of power law index (a) $n=0.75$, (b) $n=1$ 


\section{IV.CONCLUSION}

The fluid mechanics of hydrodynamic lubrication in the journal bearing operating with non-Newtonian power law lubricant was studied for incompressible, isothermal, and steady conditions. The conclusions are summarized as follows:

1. Taking into account of the cavitation model in the analysis gives a more accurate prediction with respect to the load carrying capacity and vortex development.

2. The load carrying capacity increases with increasing values of power law index $n$.

3. For power law index $n$ of 1 which corresponds to the Newtonian behavior, the discrepancies of prediction of the pressure distribution as well as the load carrying capacity between the case of "no cavitation" and the case of "with cavitation" becomes much more significant compared to the lubricant analysis with lower $n$

\section{REFERENCES}

[1] M. J. Braun and W. M. Hannon, "Cavitation formation and modelling for fluid film bearings: a review," Proc. Inst. Mech. Eng. Part J J. Eng. Tribol., vol. 224, no. 9, pp. 839-863, 2010.

[2] P. C. Mishra, "Mathematical modeling of stability in rough elliptic bore misaligned journal bearing considering thermal and nonNewtonian effects," Appl. Math. Model., vol. 37, no. 8, pp. 5896-5912, 2013.

[3] H. G. Elrod, “A Cavitation Algorithm," J. Lubr. Technol., vol. 103, no. 3, p. 350, Jul. 1981.

[4] S. K. Yadav and S. C. Sharma, "Performance of hydrostatic tilted thrust pad bearings of various recess shapes operating with nonNewtonian lubricant," Finite Elem. Anal. Des., vol. 87, pp. 43-55, 2014.

[5] J.-R. Lin, R.-F. Lu, L.-M. Chu, and C.-R. Hung, "The effects of non-Newtonian rheology in the dynamic coefficients of wide slider bearings with a secant-shaped film profile," Ind. Lubr. Tribol., vol. 65, no. 6, pp. 351-356, Sep. 2013.

[6] A. W. Pratomo, Muchammad, M. Tauviqirrahman, Jamari, and A. P. Bayuseno, "Tribological characteristic enhancement effects by polymer thickened oil in lubricated sliding contacts," in AIP Conference Proceedings 1725, 2016, vol. 020060, p. 020060.

[7] M. Tauviqirrahman, W. K. Ajie, E. Yohana, M. Muchammad, and J. Jamari, "A Study of slip position on improving the hydrodynamic lubrication performance of single-textured bearing using a mass conserving numerical approach,” Int. J. Eng. Technol., vol. 8, no. 2, pp. 913-921, 2016.

[8] C. B. Khatri and S. C. Sharma, "Influence of textured surface on the performance of non-recessed hybrid journal bearing operating with non-Newtonian lubricant," Tribol. Int., vol. 95, pp. 221-235, 2016.

[9] ANSYS, ANSYS Fluent, version 14.0: user manual. ANSYS, Inc., Canonsburg, USA., 2011.

[10] P. Zwart, A. Gerber, and T. Belamri, "A two-phase flow model for predicting cavitation dynamics," in Fifth International Conference on Multiphase Flow, Yokohama, Japan, May 30 - June 3, 2004, no. 152.

[11] Y. Qiu and M. M. Khonsari, "On the Prediction of Cavitation in Dimples Using a Mass-Conservative Algorithm,” J. Tribol., vol. 131, no. 4, p. 041702 , Oct. 2009. 